\title{
1 Projection of orthogonal tiling from the retina to the visual cortex
}

2 Jaeson Jang ${ }^{a, 1}$, Min Song ${ }^{a, b, 1}$, Gwangsu Kim ${ }^{c}$ \& Se-Bum Paik ${ }^{a, b^{*}}$

3 aDepartment of Bio and Brain Engineering, ${ }^{b}$ Program of Brain and Cognitive Engineering, 'Department of

4 Physics, Korea Advanced Institute of Science and Technology, Daejeon 34141, Republic of Korea

$5 \quad$ JJ.J. and M.S. contributed equally to this work.

6 *To whom correspondence should be addressed: Se-Bum Paik (sbpaik@kaist.ac.kr)

7

8 Keywords

9 Primary visual cortex, orientation map, ocular dominance, spatial frequency map, retinotopy, retinal 10 ganglion cell, feedforward projection

11

\section{Highlights}

13 - Orthogonal organization of visual tuning maps are observed in both V1 and the retina

14 - Cortical tuning maps are correlated with the profile of ON-OFF feedforward projections

15 - The profile of ON-OFF receptive fields varies periodically across the V1 surface

16 - Regularly structured RGC patterns initiate the orthogonal tiling of sensory modules in V1 


\section{Abstract}

19 In higher mammals, the primary visual cortex (V1) is organized into diverse tuning maps of visual

20 features such as orientation, spatial frequency and ocular dominance. The topography of these

21 maps is observed to intersect orthogonally, implying that a developmental principle for efficient

22 tiling of sensory modules may exist. However, it remains unclear how such a systematic

23 relationship among cortical tuning maps could develop. Here, we show that the orthogonal

24 organization of tuning modules already exist in retinal ganglion cell (RGC) mosaics, and that this

25 provides a blueprint of the orthogonal organization in V1. Firstly, from the analysis of multi-

26 electrode recording data in V1, we found that the ON-OFF subregion distance of receptive fields

27 varies periodically across the cortical surface, strongly correlated to ocular dominance and spatial

28 frequency in the area. Further, the ON-OFF alignment angle, that is orthogonal to the ON-OFF

29 distance, appears to correlate with orientation tuning. These suggest that the orthogonal

30 organization in V1 may originate from the spatial organization of the ON-OFF receptive fields in

31 the bottom-up projections, and this scenario was tested from analysis of the RGC mosaics data

32 in monkeys and cats. We found that the ON-OFF RGC distance and ON-OFF angle of

33 neighbouring RGCs are organized into a topographic tiling across mosaics, analogous to the

34 orthogonal intersection of cortical tuning maps. These findings suggest that the regularly

35 structured ON-OFF patterns mirrored from a retina may initiate efficient tiling of functional 36 domains in V1. 
The primary visual cortex (V1) of higher mammals is organized into a variety of functional maps of neural tuning such as preferred orientation, ocular dominance and spatial frequency. In the same cortical area, the gradient of orientation tuning intersects orthogonally with that of ocular dominance and preferred spatial frequency $(1,2)$ (Fig. 1A), resulting in an efficient tiling of diverse functional modules in V1 (2,3) (Fig. 1B). Such structural correlation between the maps implies that there may exist a common principle of developing individual functional maps (2), but how such topographical relationships among different maps could arise in V1 remains unclear.

Important clues regarding the development of the maps were found in the studies of orientation tuning in feedforward afferents. It was reported that orientation tuning in a given cortical area is predictable from the angle alignment of the local ON and OFF thalamic afferents (4), and the change of orientation tuning across the cortical surface appears to correlate with the angle between ON and OFF receptive fields $(5,6)$. These observations support the idea of the statistical wiring model, in which the orientation tuning in V1 originates from the anisotropic profile in the local structure of ON and OFF retinal ganglion cell (RGC) mosaics (7-9), mirrored by feedforward mappings from a retina - via the lateral geniculate nucleus (LGN) (10) - to V1.

If this is so, how does the orthogonal organization between orientation tuning and other tunings arise in V1? Herein, we show that such an orthogonal relationship of tuning modules already exists in retinal mosaics, and that this can be mirrored to $\mathrm{V} 1$ to initiate the clustered topography of cortical organization. Our analysis of the published $\mathrm{V} 1$ recording data measured in cats (5) (Fig. 1C) show that ocular dominance and spatial frequency in V1 are correlated to the spatial separation of ON-OFF feedforward afferents (ON-OFF distance), intersecting orthogonally with the ON-OFF alignment angle (ON-OFF angle), which is correlated to orientation tuning. Interestingly, from analysis of the RGC mosaics data in cats $(11,12)$ and monkeys $(13,14)$, we found that the same type of orthogonal organization is observed in the retinal mosaics that provide bottom-up projections to V1. By combining observations in V1

62 recording and RGC mosaic data, we demonstrate that the bottom-up projection of regularly

63 structured retinal afferents can solely initiate the efficient tiling of diverse tuning maps in the 64 visual cortex.

\section{Correlation between ON-OFF afferents and cortical tunings}

67 We analyzed recently published cat data (5), where the profile of $\mathrm{V} 1$ receptive fields and functional 68 tuning were measured together across the cortical surface (Fig. 1C, left). In this dataset, 
measurements from two electrodes parallel or perpendicular to an ocular dominance column enabled an analysis of the relationship between the cortical receptive fields and the underlying cortical tuning of the preferred orientation and ocular dominance (red and blue electrodes in Fig. 1B). We found a systematic correlation between the spatial organization of local ON-OFF receptive fields and the ocular dominance/spatial frequency tuning at each location. From the observed receptive fields, we measured the angle and the distance between the center of ON and OFF subfields (Fig. 1C, right) and examined correlations with underlying functional tunings.

As reported in previous studies (4-6), we observed that orientation tuning measured at each electrode can be predicted by the angle between ON and OFF receptive fields (ON-OFF angle; Fig. 1D). More importantly, we found that the distance between ON and OFF centers (ONOFF distance, $d$ ) periodically changes across the cortical surface (Fig. 1E, top). Notably, the spatial period of the ON-OFF distance variation was practically identical to that of the ocular dominance in the same cortical area ( $2.9 \%$ difference; estimated by fitting to a sine curve). As a result, the observed ocular dominance appeared to correlate strongly to the ON-OFF distance (Fig. 1E, bottom; Pearson correlation coefficient, $N=25, r=+0.624, P=8.54 \times 10^{-4}$ ).

We also found that the ON-OFF distance and spatial frequency tuning are systematically correlated in the same data (Fig. 1F; Pearson correlation coefficient, $N=25, r=-0.46, P=0.03$ ). We found that the deviation of the ON-OFF distance from its average $(|\Delta d|$; Fig. $1 \mathrm{~F}$, top, grey solid line) is correlated negatively with spatial frequency tuning estimated from the fast Fourier transform (FFT) analysis of $\mathrm{V} 1$ receptive fields (Fig. 1F, bottom). Considering the correlation between the ocular dominance and ON-OFF distance we observed (Fig. 1E, bottom), this topographic relationship is analogous to the previous observation that spatial frequency tuning and binocularity (1-|ocular dominance|) are correlated positively (2). These results suggest that the orthogonal organization of the spatial frequency and ocular dominance maps may be initialized by the spatial organization of the ON-OFF angle and distance in the bottom-up projections (Fig. 1G).

\section{Orthogonal organization of ON and OFF retinal ganglion cells}

If the orthogonal tiling of tuning maps in a visual cortex originates from the spatial organization of ON-OFF afferents in feedforward projections (Fig. 1G), then it is expected that the ON-OFF angle and distance are already organized orthogonally in early stages of visual pathway, and that this spatial organization is mirrored to V1. Considering the precise retinotopic projection from the 
101

102

103

104

105

106

107

108

109

110

111

112

113

114

115

116

117

118

119

120

121

122

123

124

125

126

127

128

129

130

131

132

retina to V1, we hypothesized that the orderly arrangement of ON and OFF thalamic afferents to V1 originates from the spatial distribution of ON and OFF RGCs in retinal mosaics (Fig. 2A). To validate this scenario, we used RGC mosaics data previously measured in cats (11) (Fig. 2B, left) and examined whether the angle and distance between the local ON and OFF RGC mosaics are in orthogonal organization. Based on the statistical wiring model (7), we estimated the ON-OFF angle and distance of the sampled receptive field at each position of the RGC mosaics by assuming that RGCs are locally sampled to provide feedforward afferents in the local V1 area (Fig. 2B and 2C).

We calculated the angle of the intersection between the ON-OFF angle and the distance in smoothed maps (Fig. 2D). As predicted by our scenario, we found that the angle shows a peak at approximately $90^{\circ}$ (Fig. 2E). We repeated this analysis for four sets of RGC mosaic data of different species ( $n=2$ for cat $(11,12) ; n=2$ for monkey $(13,14))$ and confirmed the orthogonal intersection between the ON-OFF angle and distance in all sets of tested data (Fig. 2F). The average distribution of all data sets showed a peak at $90^{\circ}$ significantly higher than that of the control sets with the shuffled distribution of ON-OFF angle and distance (Fig. 2G; $P=0.01$ ). These results show that the ON-OFF angle and distance in RGC mosaics intersect orthogonally across retinal space and this may provide the blueprint of orthogonal organization of functional tuning maps in V1.

\section{Discussion}

Our results suggest that the orthogonal organization among the functional maps in V1 is initiated by regularly structured retinal afferents. Previously observed topographic correlation between the orientation pinwheels and ocular dominance (13) is also readily explained by our findings. Orientation pinwheels have been observed to be preferentially located around the center of monocular regions (16). From the observed correlation between the ocular dominance and the ON-OFF distance in our analysis (Fig. 1), together with our previous model approach in which orientation pinwheels emerge where the ON-OFF distance is locally minimized or maximized (15), both the orientation pinwheels and monocular regions are expected to be seeded by the same area in the RGC mosaics, resulting in the spatial overlap in the cortical space.

Overall, our results suggest that the regularly structured periphery circuits with simple feedforward wiring can provide a common developmental framework for diverse tuning circuitry in visual cortex. 


\section{Materials and Methods}

\section{Analysis of multi-electrode recordings}

135 The multi-electrode data recorded from the cats were provided by Jose-Manuel Alonso ((5) and 136 in personal communication) for the analysis in Figure 1. To remove high-frequency spatial noise, 137 the receptive fields were filtered by a low-pass fermi filter in the frequency domain. The filter was 138 designed according to

$$
f=1 /\left(\exp \left(\frac{\omega-\mu}{K \mu}\right)+1\right)
$$

where $\mu$ is the threshold of the frequency $(4 \mathrm{cpd})$ and $K$ is the smoothness coefficient. The ONOFF angle was estimated from the Fourier transform $\Psi(\omega)$ of the receptive fields. It is defined as $\arg (\mu) / 2$, where

$$
\mu=\int|\Psi(\omega)||\omega| \exp (2 i \arg (\omega)) d \omega / \int|\Psi(\omega)| d \omega
$$

144 Samples for which either the ON or OFF subregion is entirely cancelled by the other component were excluded. The ON-OFF distance was defined as the distance between the center of the ON and OFF subregions. The location of the center of the ON and OFF subregions was defined as the strongest peak of each subregion. For multiple subregion samples, the largest subregion was chosen for analysis.

\section{Analysis of RGC mosaics}

151 To estimate the ON-OFF angle and distance seeded at each location in the RGC mosaics, we

152 modelled the cortical receptive fields by sampling the receptive fields of local ON and OFF RGCs.

153 We assumed that the RGCs are statistically wired to cortical space with a two-dimensional (2D)

154 Gaussian function with a standard deviation of $\sigma_{c o n}$. The synaptic weighting between $i^{\text {th }} \mathrm{RGC}$ and $155 j^{\text {th }}$ cortical sites, $w_{i j}$, was defined as

$$
w_{i j}=\frac{1}{\sigma_{c o n} \sqrt{2 \pi}} \exp \left(-\frac{d_{i j}^{2}}{2 \sigma_{c o n}^{2}}\right)
$$

157 where $\sigma_{\text {con }}$ was set to $0.16-0.18$ times the expected average lattice distance of OFF RGC 158 mosaics $\left(d_{O F F}\right)$. The local receptive field was calculated at each vertex of a rectangular grid with 159 a spacing distance of $0.1 d_{O F F}$ in the RGC mosaics. 
The receptive fields of RGCs and V1 neurons were defined as a center-surround model of 161 2D Gaussian and their linear sum, respectively. The standard deviation and the amplitude of the 162 surrounding region was set to 3 and 0.55 times that of the center region (17).

163

$$
\Psi_{i, R G C}= \pm\left(\frac{1}{\sigma_{R G C} \sqrt{2 \pi}} \exp \left(-\frac{r_{i}^{2}}{2 \sigma_{R G C}^{2}}\right)-\frac{0.55}{3 \sigma_{R G C} \sqrt{2 \pi}} \exp \left(-\frac{r_{i}^{2}}{18 \sigma_{R G C}^{2}}\right)\right)(+: \text { ON cell, }-: \text { OFF cell })
$$

$$
\Psi_{j, V 1}=\sum_{i} w_{i j} \cdot \Psi_{i}^{R G C}
$$
Here, $\Psi_{i, R G C}$ is the receptive field of $i^{\text {th }} \mathrm{RGC}$ and $\Psi_{j, V 1}$ is for the $j^{\text {th }}$ cortical site where $r_{i}$ is a 166 distance vector from the center of $i^{\text {th }} \mathrm{RGC}$ to each position of the visual field. The terms $\sigma_{O N, R G C}$ 167 and $\sigma_{O F F, R G C}$ were set to 0.5 times the expected average lattice distance of ON and OFF RGC 168 mosaics to satisfy the condition that the receptive fields of ON and OFF RGC mosaics cover all 169 of the visual field (18). The ON-OFF angle and distance were measured between the center-of170 mass of the modelled ON and OFF receptive fields and then the resultant maps were smoothed 171 using a 2D Gaussian kernel with sigma of $0.8-1 d_{O F F}$. 


\section{Conflict of Interest}

173 The authors declare no competing financial interests.

174

175 Acknowledgments

176 We are grateful to Jose-Manuel Alonso (State University of New York) for sharing receptive field 177 data on the cat primary visual cortex. We also thank Dario Ringach, Jose-Manuel Alonso, Matteo 178 Carandini, Daeyeol Lee and Andrea Benucci, for providing helpful comments in the early stages 179 of this study. This work was supported by the National Research Foundation of Korea (NRF) grant 180 funded by the Korea government (MSIT) (No. NRF-2019R1A2C4069863 and NRF181 2019M3E5D2A01058328) (to S.P.).

182

\section{Author contributions}

184 S.P. conceived the study. J.J., M.S. and S.P. designed the model. J.J. and M.S. performed the 185 simulations. J.J., M.S., G.K. and S.P. analyzed the data. J.J., M.S. and S.P. wrote the manuscript. 186 All authors discussed and commented on the manuscript. 


\section{References}

1. I. Nauhaus, K. J. Nielsen, A. A. Disney, E. M. Callaway, Orthogonal micro-organization of orientation and spatial frequency in primate primary visual cortex. Nat. Neurosci. 15, 1683-1690 (2012).

2. I. Nauhaus, K. J. Nielsen, E. M. Callaway, Efficient receptive field tiling in primate V1. Neuron 91, 893-904 (2016).

3. N. V. Swindale, D. Shoham, A. Grinvald, T. Bonhoeffer, M. Hübener, Visual cortex maps are optimized for uniform coverage. Nat. Neurosci. 3, 822-826 (2000).

4. J. Jin, Y. Wang, H. A. Swadlow, J. M. Alonso, Population receptive fields of ON and OFF thalamic inputs to an orientation column in visual cortex. Nat. Neurosci. 14, 232-238 (2011).

5. J. Kremkow, J. Jin, Y. Wang, J. M. Alonso, Principles underlying sensory map topography in primary visual cortex. Nature 533, 52-57 (2016).

6. K.-S. Lee, X. Huang, D. Fitzpatrick, Topology of ON and OFF inputs in visual cortex enables an invariant columnar architecture. Nature 533, 90-94 (2016).

7. D. L. Ringach, On the origin of the functional architecture of the cortex. PLoS One 2, e251 (2007).

8. S.-B. Paik, D. L. Ringach, Retinal origin of orientation maps in visual cortex. Nat. Neurosci. 14, 919-925 (2011).

9. J. Jang, S.-B. Paik, Interlayer Repulsion of Retinal Ganglion Cell Mosaics Regulates Spatial Organization of Functional Maps in the Visual Cortex. J. Neurosci. 37, 1214112152 (2017).

10. W. M. Usrey, J. B. Reppas, R. C. Reid, Specificity and strength of retinogeniculate connections. J. Neurophysiol. 82, 3527-3540 (1999).

11. X. J. Zhan, J. B. Troy, Modeling cat retinal beta-cell arrays. Vis. Neurosci. 17, 23-39 (2000).

12. H. Wässle, B. B. Boycott, R.-B. Illing, Morphology and mosaic of on- and off-beta cells in the cat retina and some functional considerations. Proc. R. Soc. B Biol. Sci. 212, 177195 (1981). 
217 13. J. L. Gauthier, et al., Receptive fields in primate retina are coordinated to sample visual space more uniformly. PLoS Biol. 7, e1000063 (2009).

219 14. M. Vidne, et al., Modeling the impact of common noise inputs on the network activity of retinal ganglion cells. J. Comput. Neurosci. 33, 97-121 (2012).

221 15. S.-B. Paik, D. L. Ringach, Link between orientation and retinotopic maps in primary visual cortex. Proc. Natl. Acad. Sci. 109, 7091-7096 (2012).

223 16. M. C. Crair, E. S. Ruthazer, D. C. Gillespie, M. P. Stryker, Ocular dominance peaks at pinwheel center singularities of the orientation map in cat visual cortex. J. Neurophysiol. 77, 3381-3385 (1997).

226 17. L. J. Croner, E. Kaplan, Receptive fields of $\mathrm{P}$ and $\mathrm{M}$ ganglion cells across the primate 227 retina. Vision Res. 35, 7-24 (1995).

228 18. V. Balasubramanian, P. Sterling, Receptive fields and functional architecture in the retina. 
A

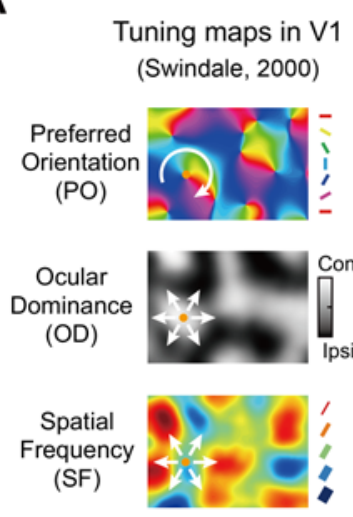

D
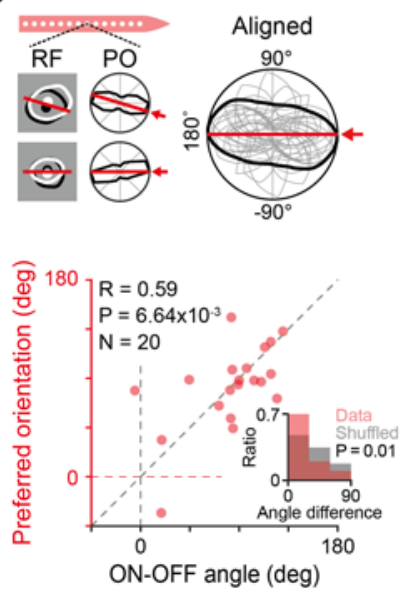

B

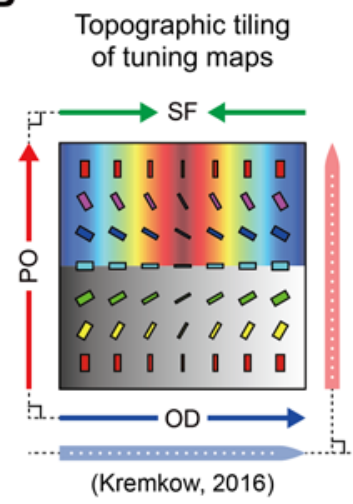

C

Intersection angle

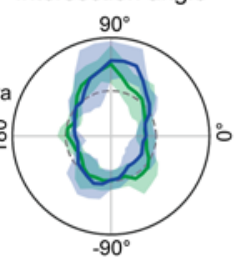

$-\mathrm{PO}-\mathrm{OD}(\mathrm{N}=7)$

- PO-SF $(\mathrm{N}=9)$

E

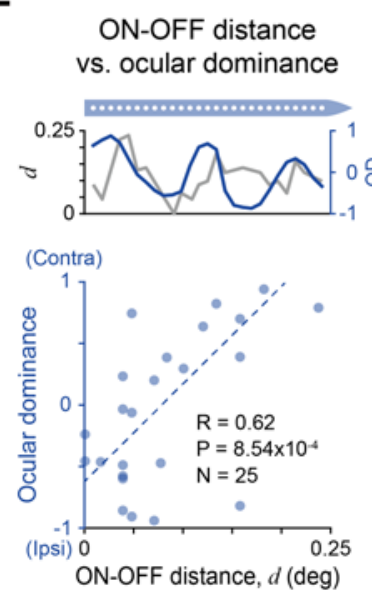

$\mathbf{F}$

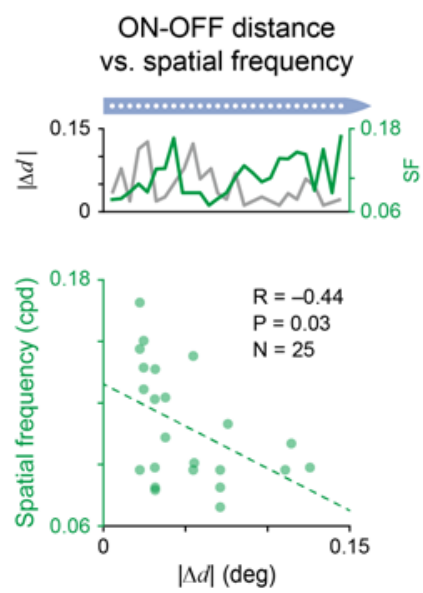

Multi-electrode recording in cat $\mathrm{V} 1$ (Kremkow, 2016)

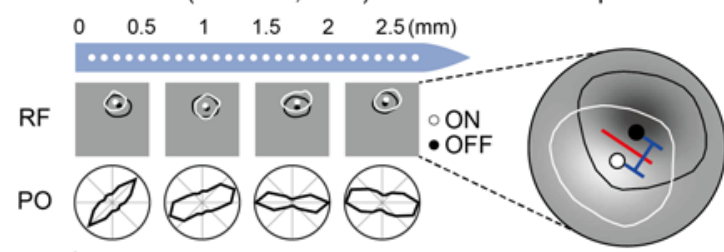

OD

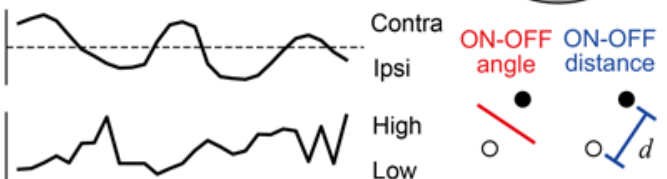

Figure 1. Topographic correlation between ON-OFF angle/distance and diverse cortical tunings

232 (A) Left: Diverse functional maps observed in V1. White solid arrows represent gradients in 233 each map. Right: Orthogonal relationship between the gradients of diverse cortical tuning maps

234 in (1-3)). $\mathrm{N}$ represents the number of map pairs examined (B) Efficient tiling of diverse function

235 tunings illustrated. (C) Left: Receptive fields and functional tunings were recorded by the

236 electrodes penetrating cat V1 (modified from Figure $2 b$ of (5)). Right: ON-OFF angle and

237 distance measured from the recorded receptive fields. (D) Top: ON-OFF angle estimated from

238 local receptive field is correlated with the orientation tuning measured at each electrode with $\mathrm{N}=$

23920 data points. Bottom: Correlation between the ON-OFF angle and the preferred orientation.

240 Inset: Angle difference between the two angles. The average difference is significantly smaller

241 than that of the shuffled pairs ( $P=0.01, N=1,000$ repeated trials). (E) Top: ON-OFF distance

242 (d) and ocular dominance vary periodically across the cortical surface. Bottom: Correlation 
243 between $d$ and ocular dominance. (F) Top: The deviation of ON-OFF distance from its average $244(|\Delta d|)$ and the spatial frequency tuning measured by the same electrode. Bottom: Negative 245 correlation between the preferred spatial frequency and $|\Delta d|$. (G) In summary, diverse cortical 246 tunings are constrained by the angle or the distance between ON and OFF feedforward 247 afferents. 
A

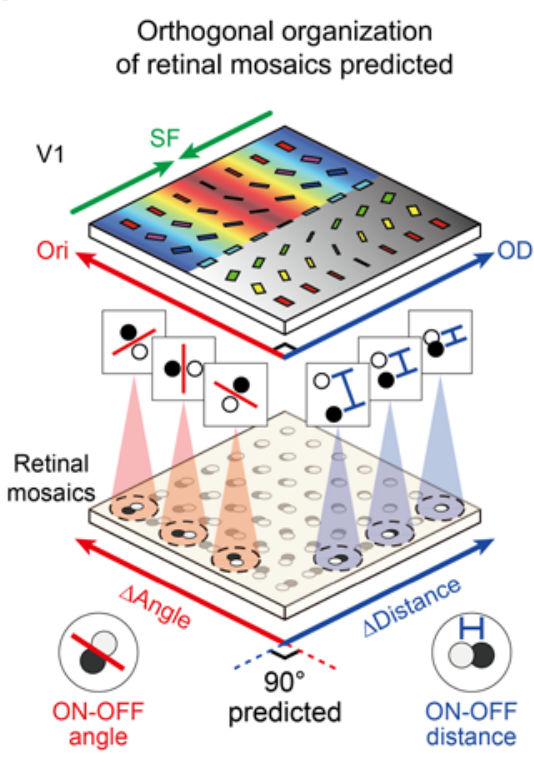

$\mathbf{F}$

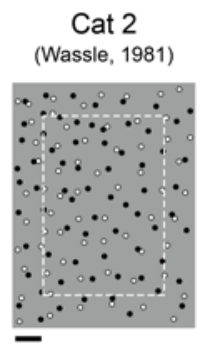

B

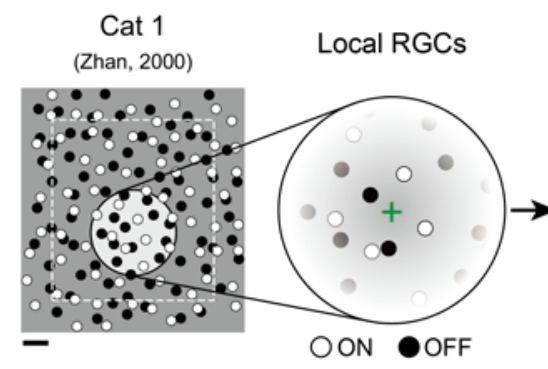

D

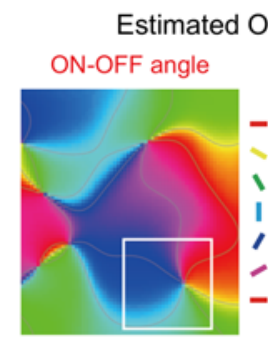

C

V1 tuning predicted

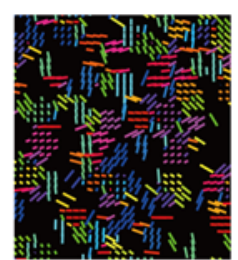

$\mathbf{E}$

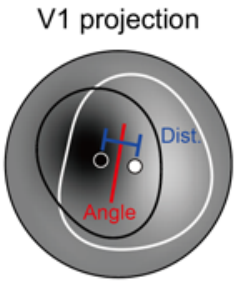

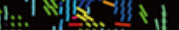

Figure 2. Orthogonal organization between ON-OFF angle and distance in RGC mosaics

249 (A) Because diverse cortical tunings change in the orthogonal direction and because each of 250 them is topographically correlated with the ON-OFF angle or distance, the ON-OFF angle and distance in RGC mosaics are also predicted to change in the orthogonal direction. (B) Angle and distance between local ON and OFF receptive fields were estimated at each position of the measured RGC mosaic data. Boundary region (width $=1 d_{O F F}$, the expected average lattice distance of OFF RGC mosaics) was excluded for accurate estimation. The RGCs are statistically selected and summed with a two-dimensional Gaussian distribution (7). (C) Map of ON-OFF angle and distance measured within the white dashed square in (B). (D) Smoothed maps of ON-OFF angle and distance. (E) Left: Contours of ON-OFF angle (colourful solid lines) and distance (grey sold line) in the white solid square in smoothed maps. Right: Orthogonal 259 intersection between gradients of ON-OFF angle and distance. Pink arrows indicate the average intersection angle. (F) Similar analyses for three other RGC mosaic data sets. (G) Averaged 
bioRxiv preprint doi: https://doi.org/10.1101/2020.02.24.963785; this version posted February 25, 2020. The copyright holder for this preprint (which was not certified by peer review) is the author/funder. All rights reserved. No reuse allowed without permission.

261 distribution of intersection angle shows a significant peak around $90^{\circ}$. Scale bar: $1 d_{O F F}$ 262 measured in each mosaic. 